\title{
On exact linesearch quasi-Newton methods for minimizing a quadratic function
}

\author{
Anders Forsgren $^{1}$ (D) $\cdot$ Tove Odland $^{1}$
}

Received: 16 September 2015 / Published online: 31 August 2017

(C) The Author(s) 2017. This article is an open access publication

\begin{abstract}
This paper concerns exact linesearch quasi-Newton methods for minimizing a quadratic function whose Hessian is positive definite. We show that by interpreting the method of conjugate gradients as a particular exact linesearch quasi-Newton method, necessary and sufficient conditions can be given for an exact linesearch quasiNewton method to generate a search direction which is parallel to that of the method of conjugate gradients. We also analyze update matrices and give a complete description of the rank-one update matrices that give search direction parallel to those of the method of conjugate gradients. In particular, we characterize the family of such symmetric rank-one update matrices that preserve positive definiteness of the quasiNewton matrix. This is in contrast to the classical symmetric-rank-one update where there is no freedom in choosing the matrix, and positive definiteness cannot be preserved. The analysis is extended to search directions that are parallel to those of the preconditioned method of conjugate gradients in a straightforward manner.
\end{abstract}

Keywords Method of conjugate gradients · Quasi-Newton method · Unconstrained quadratic program · Exact linesearch method

Research partially supported by the Swedish Research Council (VR).

$凶$ Anders Forsgren andersf@kth.se

Tove Odland odland@kth.se

1 Optimization and Systems Theory, Department of Mathematics, KTH Royal Institute of Technology, 10044 Stockholm, Sweden 


\section{Introduction}

In this paper we study the behavior of quasi-Newton methods $(\mathrm{QN})$ on an unconstrained quadratic problem of the form

$$
\underset{x \in \mathbb{R}^{n}}{\operatorname{minimize}} \frac{1}{2} x^{T} H x+c^{T} x
$$

where $H=H^{T} \succ 0$. Solving (QP) is equivalent to solving a symmetric system of linear equations $H x+c=0$. In particular, our concern is to give conditions under which a quasi-Newton method utilizing exact linesearch generates search directions that are parallel to those of the method of conjugate gradients (CG). As exact linesearch is considered, parallel search directions imply identical iterates. At iteration $k$, the $x$-iterate and the gradient $H x+c$ are denoted by $x_{k}$ and $g_{k}$ respectively. In a quasiNewton method, the search direction $p_{k}$ is computed from $B_{k} p_{k}=-g_{k}$, where $B_{k}$ is nonsingular.

We give necessary and sufficient conditions on a QN-method for this equivalence with $\mathrm{CG}$ on $(\mathrm{QP})$. This is not the first time necessary and sufficient conditions are given. In [12, Theorem 2.2], a necessary and sufficient condition is given, which is based on projections from iterations $0,1, \ldots, k-1$, allowing also the preconditioned setting to be considered. In contrast, we interpret the method of conjugate gradients as a particular quasi-Newton method and base the necessary and sufficient conditions on this observation. The result we give is thus directly based on the projection given by the method of conjugate gradients, i.e., based on quantities from iteration $k-1$ and $k$ involving one projection only.

If considering update matrices $U_{k}$ defined by $U_{k}=B_{k}-B_{k-1}$, it is well-known that, on (QP), QN using exact linesearch and an update scheme in the one-parameter Broyden family generates identical iterates to those generated by CG, see, e.g., [3, 11 , 14]. The unique rank-1 update matrix in the Broyden family is usually referred to as the SR1 update matrix, and it is determined entirely by the so-called secant condition. As a result of our equivalence result, we show that the symmetric rank-1 update matrices that give parallel search directions to CG are given by the family of update matrices on the form

$$
U_{k}=-\frac{1}{\left(\gamma_{k}-1\right) p_{k-1}^{T} g_{k-1}}\left(\gamma_{k} g_{k}-g_{k-1}\right)\left(\gamma_{k} g_{k}-g_{k-1}\right)^{T},
$$

where $\gamma_{k}$ is a free parameter. The free parameter can be seen as a relaxation of the secant condition, as the SR1 update matrix is the only matrix in our parameterized rank-1 family which satisfies this condition. We show how to choose the parameter so that positive definiteness of the quasi-Newton matrix is preserved.

To simplify the exposition, we discuss equivalence to CG, which corresponds to the initial Hessian approximation being the identity matrix in the quasi-Newton method in our analysis. We then give the corresponding results in the preconditioned setting, which corresponds to an arbitrary positive definite and symmetric initial Hessian 
approximation. For the rank-1 case, the family of symmetric update matrices take the form (1) also in the preconditioned setting.

In Sect. 2, we make a brief introduction to CG and QN. In Sect. 3, we present our results which include necessary and sufficient conditions on QN such that CG and QN generate parallel search directions. These results are specialized to update matrices in Sect. 4. In particular, in Sect. 4.1, we give the results on symmetric rank-1 update matrices. Section 5 contains a discussion on how the results would apply if the inverse of the Hessian was updated instead of the Hessian itself. In Sect. 6, the corresponding results in the preconditioned setting are stated. Finally, in Sect. 7 we make some concluding remarks.

\section{Background}

For solving (QP), we consider linesearch methods on the following form. At iteration $k$, a search direction $p_{k}$ is computed. The $x$-iterate and the gradient are updated as

$$
x_{k+1}=x_{k}+\theta_{k} p_{k}, \quad g_{k+1}=g_{k}+\theta_{k} H p_{k}, \text { for } \theta_{k}=-\frac{g_{k}^{T} p_{k}}{p_{k}^{T} H p_{k}} \text {. }
$$

The choice of steplength $\theta_{k}$ corresponds to exact linesearch, i.e., given a search direction $p_{k}$ the steplength gives the exact minimizer along $p_{k}$. This is a natural choice for (QP), as it can be done explicitly. For a given initial point $x_{0}$, the iteration process is terminated at an iteration $r$ if $g_{r}=0$, in which case $x_{r}$ is given as the optimal solution to (QP) or equivalently as the unique solution to $H x+c=0$. The method is summarized in Algorithm 1.

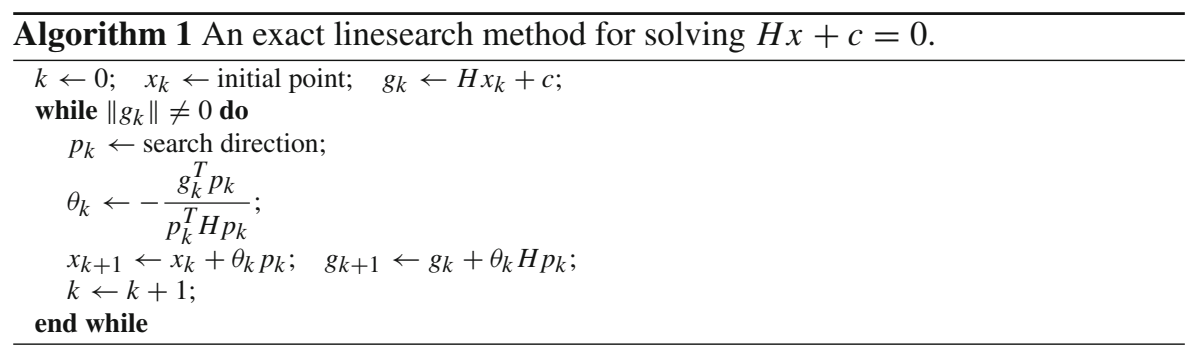

The particular linesearch method is defined by the way the search direction $p_{k}$ is obtained in each iteration $k$. Our model method is the method of conjugate gradients, CG, by Hestenes and Stiefel [10]. There are different varieties of CG, which are equivalent on $(\mathrm{QP})$. The variety we describe is referred to as the Fletcher-Reeves method of conjugate gradients, as stated in the following definition.

Definition 1 (The method of conjugate gradients $(C G)$ ) The method of conjugate gradients, CG, is the linesearch method of the form given by Algorithm 1 in which the search direction $p_{k}$ is given by $p_{k}^{C G}$, with 


$$
p_{k}^{C G}= \begin{cases}-g_{0} & \text { if } k=0, \\ -g_{k}+\frac{g_{k}^{T} g_{k}}{g_{k-1}^{T} g_{k-1}} p_{k-1}^{C G} & \text { if } k \geq 1\end{cases}
$$

For CG it holds that, for all $k, g_{k}^{T} g_{i}=0, i=0, \ldots, k-1$, so the method terminates with $g_{r}=0$ for some $r, r \leq n$, and $x_{r}$ solves (QP). In addition, it holds that $\left\{p_{k}^{C G}\right\}_{k=0}^{r-1}$ are mutually conjugate with respect to $H$. For an introduction to CG, see, e.g., $[2,15,16]$. In [5], CG is extended to general unconstrained problems. The reason for CG being our model method is that it requires one matrix-vector product $H p_{k}$ per iteration, and it terminates in $r$ iterations, with $r \leq n$.

Next we define what we will refer to as a quasi-Newton method, QN.

Definition 2 (Quasi-Newton method $(Q N)$ ) A quasi-Newton method, QN, is a linesearch method of the form given by Algorithm 1 in which the search direction $p_{k}$ is given by

$$
B_{k} p_{k}=-g_{k},
$$

where the matrix $B_{k}$ is assumed nonsingular.

Quasi-Newton methods were first suggested by Davidon, see [1], and later modified and formalized by Fletcher and Powell, see [4]. For an introduction to QN-methods, see, e.g., [7, Chapter 4].

Our interest is now to set up conditions on $B_{k}$ such that $p_{k}$ and $p_{k}^{C G}$ are parallel for all $k$, so that QN also terminates in $r$ iterations. In [6], we derived such conditions based on a sufficient condition to obtain mutually conjugate search directions. Here, we give a direct necessary and sufficient condition based on $p_{k}^{C G}$ only.

The results of the paper are derived with (CG) as the model method, which corresponds to $B_{0}=I$ in $(\mathrm{QN})$ giving $p_{0}=p_{0}^{C G}$. It is also of interest to consider the case when a symmetric positive definite matrix $M$ is given for which a preconditioned method of conjugate gradients is defined. This corresponds to $B_{0}=M$ in $(\mathrm{QN})$ giving the initial search directions identical. To simplify the exposition, we derive the results for the unpreconditioned case given by $(\mathrm{CG})$ and give the corresponding results for the preconditioned setting in Sect. 6.

\section{Necessary and sufficient conditions for QN}

In this section we give precise conditions on $B_{k}$ such that $p_{k}$ is parallel to $p_{k}^{C G}$. The main benefit of the conditions compared to previous work is that our result is based on the single iteration $k$. The dependence on the previous iterates is contained in the search direction $p_{k-1}$, and there is no need to check any condition for all the previous iterates.

In the following proposition, we give a necessary and sufficient condition on $B_{k}$ at a particular iteration $k$ to give a search direction $p_{k}$ such that $p_{k}=\delta_{k} p_{k}^{C G}$ for a scalar $\delta_{k}$. We assume that each previous search direction $p_{i}$ has been parallel to the 
corresponding search direction of $\mathrm{CG}, p_{i}^{C G}$, so that $\mathrm{QN}$ and $\mathrm{CG}$ have generated the same iterate $x_{k}$.

Proposition 1 Consider iteration $k$ of the exact linesearch method of Algorithm 1, where $1 \leq k<r$. Assume that $p_{i}=\delta_{i} p_{i}^{C G}$ with $\delta_{i} \neq 0$ for $i=0, \ldots, k-1$, where $p_{i}^{C G}, i=0, \ldots, k-1$, are the search directions of the method of conjugate gradients, as stated in Definition 1. Let $A_{k}$ be defined as

$$
A_{k}=I-\frac{1}{g_{k-1}^{T} p_{k-1}} p_{k-1} g_{k}^{T}
$$

Then,

$$
A_{k}^{-1}=I+\frac{1}{g_{k-1}^{T} p_{k-1}} p_{k-1} g_{k}^{T},
$$

and it holds that $A_{k} p_{k}^{C G}=-g_{k}$. In addition, if $p_{k}$ is given by $B_{k} p_{k}=-g_{k}$ with $B_{k}$ nonsingular, then, for any nonzero scalar $\delta_{k}$, it holds that $p_{k}=\delta_{k} p_{k}^{C G}$ if and only if

$$
B_{k} A_{k}^{-1} g_{k}=\frac{1}{\delta_{k}} g_{k},
$$

or equivalently if and only if

$$
B_{k}=A_{k}^{T} W_{k} A_{k}, \text { with } W_{k} g_{k}=\frac{1}{\delta_{k}} g_{k}, \text { for } W_{k} \text { nonsingular. }
$$

Finally, it holds that $B_{k} \succ 0$ if and only if $W_{k} \succ 0$.

Proof We have

$$
p_{k}^{C G}=-g_{k}+\frac{g_{k}^{T} g_{k}}{g_{k-1}^{T} g_{k-1}} p_{k-1}^{C G}=-\left(I+\frac{1}{g_{k-1}^{T} p_{k-1}^{C G}} p_{k-1}^{C G} g_{k}^{T}\right) g_{k}
$$

Therefore, since $p_{k-1}=\delta_{k-1} p_{k-1}^{C G}$, with $\delta_{k-1} \neq 0$, (6) gives

$$
p_{k}^{C G}=-\left(I+\frac{1}{g_{k-1}^{T} p_{k-1}} p_{k-1} g_{k}^{T}\right) g_{k}=-A_{k}^{-1} g_{k},
$$

with $A_{k}^{-1}$ given by (3). Since $g_{k}^{T} p_{k-1}=0$, multiplication of $A_{k}$ of (2) by $A_{k}^{-1}$ of (3) gives $A_{k} A_{k}^{-1}=I$, so that the stated $A_{k}$ is nonsingular with corresponding inverse $A_{k}^{-1}$. Therefore, (7) gives $A_{k} p_{k}^{C G}=-g_{k}$.

Since $B_{k}$ is assumed nonsingular and $\delta_{k} \neq 0$, it holds that $p_{k}=\delta_{k} p_{k}^{C G}$ if and only if $B_{k}\left(-\delta_{k} A_{k}^{-1} g_{k}\right)=-g_{k}$, which is equivalent to (4). Since $g_{k}^{T} p_{k-1}=0$, we obtain $A_{k}^{-T} g_{k}=g_{k}$, so that (4) is equivalent to 


$$
A_{k}^{-T} B_{k} A_{k}^{-1} g_{k}=\frac{1}{\delta_{k}} g_{k}
$$

which in turn is equivalent to (5). The final result on positive definiteness follows from the nonsingularity of $A_{k}$ by Sylvester's law of inertia, see, e.g., [8, Theorem 8.1.17].

The necessary and sufficient conditions of Proposition 1 give a straightforward way to check if a matrix $B_{k}$ is such that the corresponding QN-method and CG will generate parallel search directions. The scaling of $p_{k}^{C G}$ has a special role in our analysis and we relate $p_{k}$ to $p_{k}^{C G}$ by a scalar $\delta_{k}$. The observation that $p_{k}^{C G}$ may be written as $p_{k}^{C G}=-A_{k}^{-1} g_{k}$ for a nonsingular $A_{k}$ has been made in [12, Example 2], but the equivalence result of [12] concerns $p_{k}$ without relating to the scaling of $p_{k}^{C G}$ explicitly. Therefore, the condition of [12] involves projections on all previous iterations $0,1, \ldots, k-1$, not one single projection as we obtain. In addition, since there is no relationship to a particular scaling, there is no parameter corresponding to our $\delta_{k}$. Since such a parameter is vital for deriving later results in our paper, in particular when characterizing symmetric rank-one updates, we cannot apply the equivalence result of [12] directly. A difference in [12] is that they consider matrices $N_{k}$ that approximate $H^{-1}$ rather than matrices $B_{k}$ that approximate $H$. This is not a major difference, we discuss these issues in Sect. 5.

Note that it is not necessary to make $B_{k}-I$ increase in rank. In particular, $B_{k}=$ $A_{k}^{T} A_{k}$, corresponding to $W_{k}=I$ in Proposition 1 , is a positive-definite symmetric matrix for which $B_{k} p_{k}=-g_{k}$ gives $p_{k}=p_{k}^{C G}$.

We also note that the characterization of $B_{k}$ does only depend on information from iteration $k$ and $k-1$, since it directly inherits the properties of the method of conjugate gradients. In addition, the characterization of Proposition 1 only depends directly on quantities computed by the quasi-Newton method, the scaling of the method of conjugate gradients is not needed.

\section{Results on update matrices}

In the previous section we gave results on $B_{k}$ for a particular iteration $k$ without directly relating to any other $B_{i}, i \neq k$. It is often the case that $B_{k}$ is defined in terms of the previous matrix $B_{k-1}$ and an update matrix $U_{k}$ such that $B_{k}=B_{k-1}+U_{k}$, and that conditions are put on $U_{k}$. We have in mind a setting where information from the generated gradients is used, so that $B_{k-1}$ may be expressed as $B_{k-1}=I+V_{k}$, with $\mathcal{R}\left(V_{k}\right) \subseteq \operatorname{span}\left\{g_{0}, \ldots, g_{k-1}\right\}$. As we have in mind such a setting where in addition $B_{k}$ is symmetric, we make the assumption $B_{k-1} g_{k}=g_{k}$.

Proposition 1 can then be applied in a straightforward manner to give conditions on $U_{k}$ such that $p_{k}=\delta_{k} p_{k}^{C G}$. Note that there is a one-to-one correspondence between $U_{k}$ and $B_{k}$ given $B_{k-1}$.

Proposition 2 Consider iteration $k$ of the exact linesearch method of Algorithm 1, where $1 \leq k<r$. Assume that $p_{i}=\delta_{i} p_{i}^{C G}$ with $\delta_{i} \neq 0$ for $i=0, \ldots, k-1$, where $p_{i}^{C G}, i=0, \ldots, k-1$, are the search directions of the method of conjugate gradients, 
as stated in Definition 1. Let $B_{k-1}$ be a nonsingular matrix such that $B_{k-1} p_{k-1}=$ $-g_{k-1}$ and $B_{k-1} g_{k}=g_{k}$. Let $U_{k}=B_{k}-B_{k-1}$ and assume that $B_{k}$ and $p_{k}$ satisfy $B_{k} p_{k}=-g_{k}$, with $B_{k}$ nonsingular. Then, for any nonzero scalar $\delta_{k}$, it holds that $p_{k}=\delta_{k} p_{k}^{C G}$ if and only if

$$
U_{k}\left(g_{k}+\frac{g_{k}^{T} g_{k}}{p_{k-1}^{T} g_{k-1}} p_{k-1}\right)=\left(\frac{1}{\delta_{k}}-1\right) g_{k}+\frac{g_{k}^{T} g_{k}}{p_{k-1}^{T} g_{k-1}} g_{k-1} .
$$

Proof By assumption, $B_{k}$ is nonsingular so for $B_{k}=B_{k-1}+U_{k}$, Proposition 1 gives $p_{k}=\delta_{k} p_{k}^{C G}$ if and only if

$$
\begin{aligned}
U_{k}\left(g_{k}+\frac{g_{k}^{T} g_{k}}{p_{k-1}^{T} g_{k-1}} p_{k-1}\right) & =\frac{1}{\delta_{k}} g_{k}-B_{k-1}\left(g_{k}+\frac{g_{k}^{T} g_{k}}{p_{k-1}^{T} g_{k-1}} p_{k-1}\right) \\
& =\frac{1}{\delta_{k}} g_{k}-g_{k}+\frac{g_{k}^{T} g_{k}}{p_{k-1}^{T} g_{k-1}} g_{k-1},
\end{aligned}
$$

since $p_{k-1}$ is computed from $B_{k-1} p_{k-1}=-g_{k-1}$ and it is assumed that $B_{k-1} g_{k}=g_{k}$, so the statement of the proposition follows.

Note that in the right-hand side of (8) in Proposition 2, the component along $g_{k-1}$ is nonzero and independent of $\delta_{k}$. The component along $g_{k}$ is zero for $\delta_{k}=1$, i.e., when $p_{k}=p_{k}^{C G}$.

\subsection{Results on symmetric rank-one update matrices}

Next we consider the case when $U_{k}$ is a symmetric matrix of rank one. It is well known that the secant condition gives a unique update referred to as SR1, see, e.g., [13, Chapter 9]. The secant condition and SR1 will be discussed later in this section. Using Proposition 2 we can give a different result concerning the case when the update matrix $U_{k}$ is a symmetric matrix of rank one. In particular, we show that the family of rank-1 update matrices can be parameterized by a free parameter and that the matrix is unique for a fixed value of the parameter. This parametrization allows positive definiteness of the quasi-Newton matrix to be preserved.

The situation can be considered in two ways. First, for any given value of the scalar $\gamma_{k}$, except for three distinct values, there is a symmetric rank-1 update matrix $U_{k}$ of the form

$$
U_{k}=-\frac{1}{\left(\gamma_{k}-1\right) p_{k-1}^{T} g_{k-1}}\left(\gamma_{k} g_{k}-g_{k-1}\right)\left(\gamma_{k} g_{k}-g_{k-1}\right)^{T},
$$

for which $p_{k}=\delta_{k}\left(\gamma_{k}\right) p_{k}^{C G}$, where $\delta_{k}(\cdot)$ is a real-valued function. Second, if $p_{k}=$ $\delta_{k} p_{k}^{C G}$ is required for any given value of the scalar $\delta_{k}$, except for three distinct values, and $U_{k}$ is symmetric and of rank one, $U_{k}$ must take the form (9), with $\gamma_{k}=\gamma_{k}\left(\delta_{k}\right)$, where $\gamma_{k}(\cdot)$ is the inverse function of $\delta_{k}(\cdot)$. Consequently, except for three distinct values, there is a one-to-one correspondence between $\delta_{k}$ such that $p_{k}=\delta_{k} p_{k}^{C G}$ and $\gamma_{k}$ of the symmetric rank-1 update matrix $U_{k}$ of (9). 
The functions $\delta_{k}(\cdot)$ and $\gamma_{k}(\cdot)$ are defined in the following lemma.

Lemma 1 Consider iteration $k$ of the exact linesearch method of Algorithm 1, where $1 \leq k<r$. Assume that $p_{i}=\delta_{i} p_{i}^{C G}$ with $\delta_{i} \neq 0$ for $i=0, \ldots, k-1$, where $p_{i}^{C G}$, $i=0, \ldots, k-1$, are the search directions of the method of conjugate gradients, as stated in Definition 1. Let $\hat{\gamma}_{k}=p_{k-1}^{T} g_{k-1} / g_{k}^{T} g_{k}$. For $\delta_{k} \neq 0$ and $\gamma_{k} \neq \hat{\gamma}_{k}$, let the functions $\gamma_{k}\left(\delta_{k}\right)$ and $\delta_{k}\left(\gamma_{k}\right)$ be defined by

$$
\gamma_{k}\left(\delta_{k}\right)=-\frac{p_{k-1}^{T} g_{k-1}}{g_{k}^{T} g_{k}}\left(\frac{1}{\delta_{k}}-1\right), \quad \delta_{k}\left(\gamma_{k}\right)=\frac{1}{1-\gamma_{k} \frac{g_{k}^{T} g_{k}}{p_{k-1}^{T} g_{k-1}}}
$$

Then, the functions $\gamma_{k}(\cdot)$ and $\delta_{k}(\cdot)$ are inverses to each other.

We now characterize the symmetric rank-one update matrices that give search directions which are parallel to those of the method of conjugate gradients. In addition, we give conditions for preserving positive definiteness and a hereditary result.

Proposition 3 Consider iteration $k$ of the exact linesearch method of Algorithm 1, where $1 \leq k<r$. Assume that $p_{i}=\delta_{i} p_{i}^{C G}$ with $\delta_{i} \neq 0$ for $i=0, \ldots, k-1$, where $p_{i}^{C G}, i=0, \ldots, k-1$, are the search directions of the method of conjugate gradients, as stated in Definition 1 . Let $B_{k}$ and $p_{k}$ satisfy $B_{k} p_{k}=-g_{k}$, and let $B_{k-1}$ be a nonsingular matrix such that $B_{k-1} p_{k-1}=-g_{k-1}$ and $B_{k-1} g_{k}=g_{k}$. In addition, let $\gamma_{k}(\cdot), \delta_{k}(\cdot)$ and $\hat{\gamma}_{k}$ be given by Lemma 1 .

For any scalar $\gamma_{k}$, except $\gamma_{k}=0, \gamma_{k}=\hat{\gamma}_{k}$ and $\gamma_{k}=1$, let $B_{k}$ be defined by

$$
B_{k}=B_{k-1}-\frac{1}{\left(\gamma_{k}-1\right) p_{k-1}^{T} g_{k-1}}\left(\gamma_{k} g_{k}-g_{k-1}\right)\left(\gamma_{k} g_{k}-g_{k-1}\right)^{T}
$$

Then, $B_{k}$ is nonsingular and $p_{k}=\delta_{k} p_{k}^{C G}$ for $\delta_{k}=\delta_{k}\left(\gamma_{k}\right)$.

Conversely, for any scalar $\delta_{k}$, except $\delta_{k}=0, \delta_{k}=\delta_{k}(1)$ and $\delta_{k}=1$, assume that $p_{k}=\delta_{k} p_{k}^{C G}$ and assume that $B_{k}-B_{k-1}$ is symmetric and of rank one. Then, $B_{k}$ is a nonsingular matrix given by (10) for $\gamma_{k}=\gamma_{k}\left(\delta_{k}\right)$.

If, in addition, $B_{k-1}=B_{k-1}^{T} \succ 0$, then $B_{k}$ defined by (10) satisfies $B_{k} \succ 0$ if and only if $\gamma_{k}>1$ or $\hat{\gamma}_{k}<\gamma_{k}<0$, or equivalently if and only if $\gamma_{k}=\gamma_{k}\left(\delta_{k}\right)$ for $0<\delta_{k}<\delta_{k}(1)$ or $\delta_{k}>1$.

Finally, if $B_{i} p_{i}=-g_{i}, i=0, \ldots, k$, with $B_{0}=I$ and if, for $i=1, \ldots, k, B_{i-1}$ is updated to $B_{i}$ according to (10) for $\gamma_{i}$ such that $\gamma_{i} \neq 0, \gamma_{i} \neq \hat{\gamma}_{i}$ and $\gamma_{i} \neq 1$, then

$$
B_{k} p_{i}=\frac{\gamma_{i+1} \theta_{i}}{\gamma_{i+1}-1} H p_{i}, \quad i=0, \ldots, k-1
$$

Proof Let $U_{k}=B_{k}-B_{k-1}$. If $U_{k}$ is symmetric and of rank one, we may write $U_{k}=\beta_{k} u_{k} u_{k}^{T}$, where $\beta_{k}$ is a scalar and $u_{k}$ is a vector in $\mathbb{R}^{n}$, both to be determined. If $B_{k}$ is nonsingular and $\delta_{k} \neq 0$, Proposition 2 shows that $p_{k}=\delta_{k} p_{k}^{C G}$ if and only if 


$$
\beta_{k} u_{k} u_{k}^{T} A_{k}^{-1} g_{k}=\left(\frac{1}{\delta_{k}}-1\right) g_{k}+\frac{g_{k}^{T} g_{k}}{p_{k-1}^{T} g_{k-1}} g_{k-1}=-\frac{g_{k}^{T} g_{k}}{p_{k-1}^{T} g_{k-1}}\left(\gamma_{k} g_{k}-g_{k-1}\right),
$$

with $\gamma_{k}=\gamma_{k}\left(\delta_{k}\right)$ given by Lemma 1. Throughout the proof, assume that $\delta_{k} \notin$ $\left\{0, \delta_{k}(1), 1\right\}$ and $\gamma_{k} \notin\left\{0, \hat{\gamma}_{k}, 1\right\}$, which is assumed in the statement of the Proposition. Then, Lemma 1 shows that there is a one-to-one correspondence between $\delta_{k}$ and $\gamma_{k}$. Hence, (12) may be considered for either $\delta_{k}$ or $\gamma_{k}$. We choose $\gamma_{k}$ for ease of notation.

We first assume that $B_{k}$ is nonsingular, and verify that this is the case later in the proof. For $B_{k}$ nonsingular, it follows from (12) that $u_{k}$ will be equal to the right-hand side vector up to some arbitrary non-zero scaling. Let

$$
u_{k}=\gamma_{k} g_{k}-g_{k-1}
$$

The scaling of $u_{k}$ will be reflected in $\beta_{k}$ by insertion into (12) as

$$
\beta_{k}\left(\gamma_{k} g_{k}-g_{k-1}\right)^{T}\left(g_{k}+\frac{g_{k}^{T} g_{k}}{p_{k-1}^{T} g_{k-1}} p_{k-1}\right)=\beta_{k}\left(\gamma_{k}-1\right) g_{k}^{T} g_{k}=-\frac{g_{k}^{T} g_{k}}{p_{k-1}^{T} g_{k-1}},
$$

so that

$$
\beta_{k}=-\frac{1}{\left(\gamma_{k}-1\right) p_{k-1}^{T} g_{k-1}} .
$$

Note that (14) is well defined as $\gamma_{k} \neq 1$ is assumed. A combination of (12), (13) and (14) gives $B_{k}$ expressed as in (10).

It remains to show that $B_{k}$ is nonsingular. It follows from (10) that

$$
B_{k}=B_{k-1}\left(I-\frac{1}{\left(\gamma_{k}-1\right) p_{k-1}^{T} g_{k-1}}\left(\gamma_{k} B_{k-1}^{-1} g_{k}-B_{k-1}^{-1} g_{k-1}\right)\left(\gamma_{k} g_{k}-g_{k-1}\right)^{T}\right)
$$

so that

$$
\operatorname{det}\left(B_{k}\right)=\operatorname{det}\left(B_{k-1}\right) \eta_{k}
$$

with

$$
\begin{aligned}
\eta_{k} & =1-\frac{\left(\gamma_{k} B_{k-1}^{-1} g_{k}-B_{k-1}^{-1} g_{k-1}\right)^{T}\left(\gamma_{k} g_{k}-g_{k-1}\right)}{\left(\gamma_{k}-1\right) p_{k-1}^{T} g_{k-1}} \\
& =1-\frac{\gamma_{k}^{2} g_{k}^{T} g_{k}-p_{k-1}^{T} g_{k-1}}{\left(\gamma_{k}-1\right) p_{k-1}^{T} g_{k-1}}=\frac{-\gamma_{k}\left(\gamma_{k} g_{k}^{T} g_{k}-p_{k-1}^{T} g_{k-1}\right)}{\left(\gamma_{k}-1\right) p_{k-1}^{T} g_{k-1}} \\
& =\frac{-\gamma_{k}\left(\gamma_{k}-\hat{\gamma}_{k}\right)}{\left(\gamma_{k}-1\right) \hat{\gamma}_{k}}
\end{aligned}
$$

since $B_{k-1} g_{k}=g_{k}, B_{k-1} p_{k-1}=-g_{k-1}$ and $g_{k}^{T} p_{k-1}=0$, with $\hat{\gamma}_{k}$ given by Lemma 1 . Hence, since $B_{k-1}$ is assumed nonsingular, a combination of (15) and (16) shows that 
nonsingularity of $B_{k}$ is equivalent to $\eta_{k} \neq 0$, i.e., $\gamma_{k} \neq 0$ and $\gamma_{k} \neq \hat{\gamma}_{k}$, which is exactly what is assumed.

To prove the result on positive definiteness, assume that $B_{k-1}=B_{k-1}^{T} \succ 0$. In this case, since $B_{k-1}$ and $B_{k}$ differ by a symmetric rank-1 matrix, $B_{k}$ can have at most one nonpositive eigenvalue, see, e.g., [8, Theorem 8.1.8]. Therefore, (15) shows that positive definiteness of $B_{k}$ is equivalent to $\eta_{k}>0$. Note that $B_{k-1} \succ 0$ implies $p_{k-1}^{T} g_{k-1}=-p_{k-1}^{T} B_{k-1}^{-1} p_{k-1}<0$, which in turn gives $\hat{\gamma}_{k}<0$. We may now examine (16) to see what values of $\gamma_{k}$ that give $\eta_{k}>0$. The numerator of (16) is positive for $\hat{\gamma}_{k}<\gamma_{k}<0$ and negative for $\gamma_{k}<\hat{\gamma}_{k}$ and $\gamma_{k}>0$. The denominator of (16) is positive for $\gamma_{k}<1$ and negative for $\gamma_{k}>1$. We conclude that $\eta_{k}>0$ if and only if $\hat{\gamma}_{k}<\gamma_{k}<0$ or $\gamma_{k}>1$, which by Lemma 1 is equivalent to $0<\delta_{k}<\delta_{k}(1)$ or $\delta_{k}>1$.

To prove the final hereditary result, assume that $B_{i} p_{i}=-g_{i}, i=0, \ldots, k$, with $B_{0}=I$ and assume that $B_{i-1}$ is updated to $B_{i}$ according to (10) for $\gamma_{i}$ such that $\gamma_{i} \neq 0, \gamma_{i} \neq \hat{\gamma}_{i}$ and $\gamma_{i} \neq 1$. Then, for a given $i, 0<i<k, k$ may be replaced by $i+1$ in (10), which gives

$$
\begin{aligned}
B_{i+1} p_{i} & =B_{i} p_{i}-\frac{1}{\left(\gamma_{i+1}-1\right) p_{i}^{T} g_{i}}\left(\gamma_{i+1} g_{i+1}-g_{i}\right)\left(\gamma_{i+1} g_{i+1}-g_{i}\right)^{T} p_{i} \\
& =-g_{i}+\frac{1}{\gamma_{i+1}-1}\left(\gamma_{i+1} g_{i+1}-g_{i}\right)=\frac{\gamma_{i+1}}{\gamma_{i+1}-1}\left(g_{i+1}-g_{i}\right) \\
& =\frac{\gamma_{i+1} \theta_{i}}{\gamma_{i+1}-1} H p_{i}
\end{aligned}
$$

where the identities $B_{i} p_{i}=-g_{i}, g_{i+1}^{T} p_{i}=0$ and $g_{i+1}-g_{i}=\theta_{i} H p_{i}$ have been used. Finally, (10) gives $B_{j} p_{i}=B_{i+1} p_{i}$ for $j=i+2, \ldots, k$, since $g_{j}^{T} p_{i}=0$ for $j \geq i+1$. Consequently, $B_{k} p_{i}=B_{i+1} p_{i}$, with $B_{i+1} p_{i}$ given by (17), proving (11).

Note that there are two ways in which positive definiteness of a symmetric $B_{k-1}$ may be preserved in a symmetric rank-one update. The first one, $\gamma_{k}>1$, or equivalently $0<\delta_{k}<\delta_{k}(1)$, is straightforward, since it corresponds to $U_{k} \succeq 0$. The second one, $\hat{\gamma}_{k}<\gamma_{k}<0$, or equivalently $\delta_{k}>1$, is less straightforward. The corresponding $U_{k}$ is negative semidefinite, but still the resulting $B_{k}$ is positive definite.

Proposition 3 gives precise conditions for which rank-one matrices that give a corresponding update matrix that preserves positive definiteness and gives search directions parallel to the method of conjugate gradients. We have the freedom to choose $\gamma_{k}$ or $\delta_{k}$ appropriately. This can be compared to SR1, the symmetric rank-one update scheme uniquely defined by the secant condition

$$
B_{k} s_{k-1}=y_{k}, \text { for } s_{k-1}=\theta_{k-1} p_{k-1}, \quad y_{k}=g_{k}-g_{k-1} .
$$

By writing $U_{k}=B_{k}-B_{k-1}$, the secant condition gives a requirement on $U_{k}$ as

$$
U_{k} s_{k-1}=y_{k}-B_{k-1} s_{k-1},
$$

which for $U_{k}$ symmetric and of rank one gives the SR1 update matrix $U_{k}^{S R 1}$ on the form 


$$
U_{k}^{S R 1}=\frac{1}{s_{k-1}^{T}\left(y_{k}-B_{k} s_{k-1}\right)}\left(y_{k}-B_{k} s_{k-1}\right)\left(y_{k}-B_{k} s_{k-1}\right)^{T},
$$

see, e.g., [13, Chapter 9]. Since $B_{k-1} p_{k-1}=-g_{k-1}$ holds by the definition of the quasi-Newton method, we may use the definitions of $s_{k-1}$ and $y_{k}$ of (18) to rewrite $U_{k}^{S R 1}$ of (20) as

$U_{k}^{S R 1}=\frac{1}{\theta_{k-1} p_{k-1}^{T}\left(g_{k}-\left(1-\theta_{k-1}\right) g_{k-1}\right.}\left(g_{k}-\left(1-\theta_{k-1}\right) g_{k-1}\right)\left(g_{k}-\left(1-\theta_{k-1}\right) g_{k-1}\right)^{T}$.

Since exact linesearch is performed in our case, it holds that $p_{k-1}^{T} g_{k}=0$, so that $U_{k}^{S R 1}$ takes the form

$$
\begin{aligned}
U_{k}^{S R 1} & =\frac{-1}{\theta_{k-1}\left(1-\theta_{k-1}\right) p_{k-1}^{T} g_{k-1}}\left(g_{k}-\left(1-\theta_{k-1}\right) g_{k-1}\right)\left(g_{k}-\left(1-\theta_{k-1}\right) g_{k-1}\right)^{T} \\
& =\frac{-\left(1-\theta_{k-1}\right)}{\theta_{k-1} p_{k-1}^{T} g_{k-1}}\left(\frac{1}{1-\theta_{k-1}} g_{k}-g_{k-1}\right)\left(\frac{1}{1-\theta_{k-1}} g_{k}-g_{k-1}\right)^{T},
\end{aligned}
$$

where in the last step, a scaling of the rank-1 vector by a factor $1 /\left(1-\theta_{k-1}\right)$ has been made. A comparison of (10) and (21) shows that the SR1 update is the particular member of the family of symmetric rank-1 updates given by Proposition 3 for which $\gamma_{k}=1 /\left(1-\theta_{k-1}\right)$. In particular, for $\theta_{k-1}=1$, SR 1 is not well defined. In addition, as there is no freedom in choosing the rank-one matrix for SR1, there is no way to ensure $B_{k} \succ 0$ even if $B_{k-1}=B_{k-1}^{T} \succ 0$. Note that the condition on $B_{k}$ of (18) giving a condition on $U_{k}$ of (19) and a unique symmetric rank-1 $U_{k}$ of (20) is analogous to our condition on $B_{k}$ of Proposition 1 for a fixed $\delta_{k}$ giving a condition on $U_{k}$ of Proposition 2 and a unique rank-1 $U_{k}$ of Proposition 3.

Example 1 illustrates the SR1 update and another rank-1 update of Proposition 3 which preserves positive definiteness. The $H$ and $c$ of the example are parameterized by a positive scalar $\phi$. We obtain $\theta_{0}=2 /(3 \phi)$, so by selecting $\phi=2 / 3$, it follows that $\theta_{0}=1$ and the SR1 update becomes undefined. By selecting $\phi$ slightly smaller than $2 / 3$, for example 0.65 , we obtain $\theta_{0}$ slightly larger than one $\left(\theta_{0}=40 / 39\right)$, so that $\gamma_{1}=-39$ and the corresponding $\delta_{1}$ is negative $\left(\delta_{1}=-3 / 10\right)$. Consequently, $B_{1}^{S R 1}$ is indefinite and the corresponding $p_{1}$ is an ascent direction. For comparison, the rank-1 update of Proposition 3 is given for $\delta_{1}=2$, which preserves positive definiteness. As can be seen from (10), the rank-1 update of Proposition 3 is independent of $\phi$.

Example 1 For a positive parameter $\phi$, consider the example

$$
H=\phi\left(\begin{array}{ll}
2 & 0 \\
0 & 1
\end{array}\right), \quad c=\phi\left(\begin{array}{l}
-1 \\
-1
\end{array}\right), \quad B_{0}=\left(\begin{array}{ll}
1 & 0 \\
0 & 1
\end{array}\right), \quad x_{0}=\left(\begin{array}{l}
0 \\
0
\end{array}\right)
$$

for which

$$
x_{1}=\left(\begin{array}{c}
\frac{2}{3} \\
\frac{2}{3}
\end{array}\right), \quad x_{2}=\left(\begin{array}{c}
\frac{1}{2} \\
1
\end{array}\right) \text {. }
$$


Then

$$
\begin{array}{llll}
\phi=\frac{2}{3} & \Longrightarrow & B_{1}^{S R 1} \text { undefined } & B_{1}^{\delta_{1}=2}=\frac{1}{44}\left(\begin{array}{rr}
43 & 5 \\
5 & 19
\end{array}\right) \\
\phi=\frac{65}{100} & \Longrightarrow & B_{1}^{S R 1}=\frac{1}{20}\left(\begin{array}{rr}
-16 & 42 \\
42 & -29
\end{array}\right) & B_{1}^{\delta_{1}=2}=\frac{1}{44}\left(\begin{array}{rr}
43 & 5 \\
5 & 19
\end{array}\right)
\end{array}
$$

Note that the numerical values and the dimension of Example 1 are not important. For a given quadratic problem, there will always exist a particular positive scaling such that the resulting $B_{1}^{S R 1}$ is undefined.

\section{On the approximation of the Hessian}

The results of the present manuscript have been written based on the search directions of the method of conjugate gradients. The reason for doing so is that it allows a direct treatment of $B_{k}$, and there is no need to focus on the update matrix $B_{k}-B_{k-1}$. This is the choice of the authors, but other choices are of course possible.

The results are stated for a matrix $B_{k}$ that approximates the Hessian $H$. We prefer to think of the quasi-Newton method in this way, but there would be little difference if one instead stated the results for a matrix $N_{k}$ that approximates $H^{-1}$, which is done for example in [12]. The search direction $p_{k}$ would then be defined by $p_{k}=-N_{k} g_{k}$ rather than by $B_{k} p_{k}=-g_{k}$ and conditions would be imposed on $N_{k}$ rather than on $B_{k}$. Proposition 1 could be equivalently stated using $N_{k}$ as the approximation of $H^{-1}$. Then, the counterparts of (4) and (5) would read

$$
N_{k} g_{k}=\delta_{k} A_{k}^{-1} g_{k} \text { and } A_{k} N_{k} A_{k}^{T} g_{k}=\delta_{k} g_{k}
$$

When considering update matrices, with $V_{k}=N_{k}-N_{k-1}$, the counterpart of the update formula (8) of Proposition 2 would read

$$
V_{k} g_{k}=\delta_{k} A_{k}^{-1} g_{k}-N_{k-1} g_{k}=\left(\delta_{k}-1\right) g_{k}+\delta_{k} \frac{g_{k}^{T} g_{k}}{g_{k-1}^{T} p_{k-1}} p_{k-1} .
$$

For the rank-one case, the update matrix is unique for a given $\delta_{k}$, and (22) gives

$$
N_{k}=N_{k-1}-\frac{1}{\gamma_{k}\left(\gamma_{k} g_{k}^{T} g_{k}-p_{k-1}^{T} g_{k-1}\right)}\left(\gamma_{k} g_{k}+p_{k-1}\right)\left(\gamma_{k} g_{k}+p_{k-1}\right)^{T} \text {, }
$$

with $\gamma_{k}=\gamma_{k}\left(\delta_{k}\right)$ of Lemma 1. The uniqueness of the update implies that if $N_{k-1}=$ $B_{k-1}^{-1}$, then $N_{k}=B_{k}^{-1}$ and (23) follows from (10) by the Sherman-Morrison formula.

As for the rank-one case, in light of the results of Sect. 4.1, one of the referees has pointed out that the parametrization given by $\delta_{k}$ can be replaced by a different parametrization. The secant condition $\theta_{k-1} B_{k} p_{k-1}=g_{k}-g_{k-1}$ and its counterpart on the inverse $\theta_{k-1} p_{k-1}=N_{k}\left(g_{k}-g_{k-1}\right)$ may be relaxed by a parameter $\lambda_{k}$ so that 
$\lambda_{k} \theta_{k-1} B_{k} p_{k-1}=g_{k}-g_{k-1}$ and $\lambda_{k} \theta_{k-1} p_{k-1}=N_{k}\left(g_{k}-g_{k-1}\right)$ respectively. For the updates, we obtain

$$
\begin{aligned}
\lambda_{k} \theta_{k-1} U_{k} p_{k} & =g_{k}-\left(1-\lambda_{k} \theta_{k-1}\right) g_{k-1} \text { and } \\
V_{k}\left(g_{k}-g_{k-1}\right) & =-g_{k}-\left(1-\lambda_{k} \theta_{k-1}\right) p_{k-1},
\end{aligned}
$$

since $B_{k-1} p_{k-1}=-g_{k-1}, N_{k-1} g_{k}=g_{k}$ and $N_{k-1} g_{k-1}=-p_{k-1}$. If having read the previous sections of this paper, we would see that $p_{k}=\delta_{k} p_{k}^{C G}$, where we can relate $\gamma_{k}$ to $\lambda_{k}$ by $\gamma_{k}=1 /\left(1-\lambda_{k} \theta_{k-1}\right)$, by comparing the right-hand side vector of (24a) to the rank-one vector of Proposition 3 or comparing the right-hand side vector of (24b) to the rank-one vector of (23). The corresponding relationship to $\delta_{k}$ is given by Lemma 1 . An alternative to reading the previous sections of this paper, however, would be to say that $\lambda_{k}=1$ corresponds to the SR1 update, and $\lambda_{k}=0$ corresponds to the conjugate projection update [3, Eq. (4.1.10)]. They are considered in the update of the inverse and are both known to give $p_{k}$ parallel to $p_{k}^{C G}$ if $N_{0}=I$. By replacing $\gamma_{k}$ by $1 /\left(1-\lambda_{k} \theta_{k-1}\right)$, one could show that a rank-1 matrix of the form (9) would give $p_{k}$ parallel to $p_{k}^{C G}$ using induction similar to what is done in [3, Theorem 3.4.1] and give conditions on preserving positive definiteness on $\lambda_{k}$. This would, however, not show that there is no other family of rank-1 updates giving $p_{k}$ parallel to $p_{k}^{C G}$. We prefer to give a direct proof based on our result of Proposition 1, as we from there get both necessary and sufficient conditions.

\section{Preconditioning}

Our results have been derived in the setting of CG, which corresponds to $B_{0}=I$ in QN giving the initial search directions identical. In this section, we give the analogous results in a preconditioned setting. In the preconditioned method of conjugate gradients, there is a positive definite symmetric matrix $M$, providing an estimate of $H$. For the quasi-Newton method, this will correspond to $B_{0}=M$ giving the initial search directions identical.

The preconditioned method of conjugate gradient takes the following form. If the Cholesky factor of $M$ is denoted by $L$, so that $M=L L^{T}$, then the method of conjugate gradients is applied to

$$
L^{-1} H L^{-T \widehat{x}}+L^{-1} c=0,
$$

for $\widehat{x}=L^{T} x$, see, e.g., [15, Chapter 9.2]. Letting "hat" be associated with quantities of (25), we obtain $\widehat{p}=L^{T} p$ and $\widehat{g}=L^{-1} g$. Since $\widehat{p}$ is associated with a "usual" unpreconditioned system, we write $\widehat{p}^{C G}$, and since $p$ is associated with a preconditioned system, we write $p^{P C G}$, so that $\widehat{p}^{C G}=L^{T} p^{P C G}$. It is straightforward to use these relations to derive the result analogous to those given in the the previous sections also for the preconditioned system.

Definition 3 (The preconditioned method of conjugate gradients (PCG)) For a positive definite symmetric $n \times n$ matrix $M$, the preconditioned method of conjugate gradients, PCG, is the linesearch method of the form given by Algorithm 1 in which the search direction $p_{k}$ is given by $p_{k}^{P C G}$, with 


$$
p_{k}^{P C G}= \begin{cases}-M^{-1} g_{0} & \text { if } k=0 \\ -M^{-1} g_{k}+\frac{g_{k}^{T} M^{-1} g_{k}}{g_{k-1}^{T} M^{-1} g_{k-1}} p_{k-1}^{P C G} & \text { if } k \geq 1\end{cases}
$$

For PCG it holds that, for all $k, g_{k}^{T} M^{-1} g_{i}=0, i=0, \ldots, k-1$, so the method terminates with $g_{r}=0$ for some $r, r \leq n$, and $x_{r}$ solves (QP). In addition, it holds that $\left\{p_{k}^{P C G}\right\}_{k=0}^{r-1}$ are mutually conjugate with respect to $H$.

Proposition 4 Consider iteration $k$ of the exact linesearch method of Algorithm 1 , where $1 \leq k<r$. Assume that $p_{i}=\delta_{i} p_{i}^{P C G}$ with $\delta_{i} \neq 0$ for $i=0, \ldots, k-1$, where $p_{i}^{P C G}, i=0, \ldots, k-1$, are the search directions of the preconditioned method of conjugate gradients, as stated in Definition 3. Let $A_{k}$ be defined as

$$
A_{k}=I-\frac{1}{g_{k-1}^{T} p_{k-1}} p_{k-1} g_{k}^{T}
$$

Then,

$$
A_{k}^{-1}=I+\frac{1}{g_{k-1}^{T} p_{k-1}} p_{k-1} g_{k}^{T},
$$

and it holds that $M A_{k} p_{k}^{P C G}=-g_{k}$. In addition, if $p_{k}$ is given by $B_{k} p_{k}=-g_{k}$ with $B_{k}$ nonsingular, then, for any nonzero scalar $\delta_{k}$, it holds that $p_{k}=\delta_{k} p_{k}^{P C G}$ if and only if

$$
B_{k} A_{k}^{-1} M^{-1} g_{k}=\frac{1}{\delta_{k}} g_{k},
$$

or equivalently if and only if

$$
B_{k}=A_{k}^{T} W_{k} A_{k}, \text { with } W_{k} M^{-1} g_{k}=\frac{1}{\delta_{k}} g_{k}, \text { for } W_{k} \text { nonsingular. }
$$

Finally, it holds that $B_{k} \succ 0$ if and only if $W_{k} \succ 0$.

In particular, $B_{k}=A_{k}^{T} M A_{k}$, corresponding to $W_{k}=M$ in Proposition 4 , is a positive-definite symmetric matrix for which $B_{k} p_{k}=-g_{k}$ gives $p_{k}=p_{k}^{P C G}$.

Proposition 5 Consider iteration $k$ of the exact linesearch method of Algorithm 1, where $1 \leq k<r$. Assume that $p_{i}=\delta_{i} p_{i}^{P C G}$ with $\delta_{i} \neq 0$ for $i=0, \ldots, k-1$, where $p_{i}^{P C G}, i=0, \ldots, k-1$, are the search directions of the preconditioned method of conjugate gradients using a positive definite symmetric preconditioning matrix $M$, as stated in Definition 3. Let $B_{k-1}$ be a nonsingular matrix such that $B_{k-1} p_{k-1}=-g_{k-1}$ and $B_{k-1} M^{-1} g_{k}=g_{k}$. Let $U_{k}=B_{k}-B_{k-1}$ and assume that $B_{k}$ and $p_{k}$ satisfy $B_{k} p_{k}=-g_{k}$, with $B_{k}$ nonsingular. Then, for any nonzero scalar $\delta_{k}$, it holds that $p_{k}=\delta_{k} p_{k}^{P C G}$ if and only if

$$
U_{k}\left(M^{-1} g_{k}+\frac{g_{k}^{T} M^{-1} g_{k}}{p_{k-1}^{T} g_{k-1}} p_{k-1}\right)=\left(\frac{1}{\delta_{k}}-1\right) g_{k}+\frac{g_{k}^{T} M^{-1} g_{k}}{p_{k-1}^{T} g_{k-1}} g_{k-1} \text {. }
$$


Lemma 2 Consider iteration $k$ of the exact linesearch method of Algorithm 1, where $1 \leq k<r$. Assume that $p_{i}=\delta_{i} p_{i}^{P C G}$ with $\delta_{i} \neq 0$ for $i=0, \ldots, k-1$, where $p_{i}^{P C G}, i=0, \ldots, k-1$, are the search directions of the preconditioned method of conjugate gradients using a positive definite symmetric preconditioning matrix $M$, as stated in Definition 3. Let $\hat{\gamma}_{k}=p_{k-1}^{T} g_{k-1} / g_{k}^{T} M^{-1} g_{k}$. For $\delta_{k} \neq 0$ and $\gamma_{k} \neq \hat{\gamma}_{k}$, let the functions $\gamma_{k}\left(\delta_{k}\right)$ and $\delta_{k}\left(\gamma_{k}\right)$ be defined by

$$
\gamma_{k}\left(\delta_{k}\right)=-\frac{p_{k-1}^{T} g_{k-1}}{g_{k}^{T} M^{-1} g_{k}}\left(\frac{1}{\delta_{k}}-1\right), \quad \delta_{k}\left(\gamma_{k}\right)=\frac{1}{1-\gamma_{k} \frac{g_{k}^{T} M^{-1} g_{k}}{p_{k-1}^{T} g_{k-1}}} .
$$

Then, the functions $\gamma_{k}(\cdot)$ and $\delta_{k}(\cdot)$ are inverses to each other.

Proposition 6 Consider iteration $k$ of the exact linesearch method of Algorithm 1, where $1 \leq k<r$. Assume that $p_{i}=\delta_{i} p_{i}^{P C G}$ with $\delta_{i} \neq 0$ for $i=0, \ldots, k-1$, where $p_{i}^{P C G}, i=0, \ldots, k-1$, are the search directions of the preconditioned method of conjugate gradients using a positive definite symmetric preconditioning matrix $M$, as stated in Definition 3. Let $B_{k}$ and $p_{k}$ satisfy $B_{k} p_{k}=-g_{k}$, and let $B_{k-1}$ be anonsingular matrix such that $B_{k-1} p_{k-1}=-g_{k-1}$ and $B_{k-1} M^{-1} g_{k}=g_{k}$. In addition, let $\gamma_{k}(\cdot)$, $\delta_{k}(\cdot)$ and $\hat{\gamma}_{k}$ be given by Lemma 2 .

For any scalar $\gamma_{k}$, except $\gamma_{k}=0, \gamma_{k}=\hat{\gamma}_{k}$ and $\gamma_{k}=1$, let $B_{k}$ be defined by

$$
B_{k}=B_{k-1}-\frac{1}{\left(\gamma_{k}-1\right) p_{k-1}^{T} g_{k-1}}\left(\gamma_{k} g_{k}-g_{k-1}\right)\left(\gamma_{k} g_{k}-g_{k-1}\right)^{T} .
$$

Then, $B_{k}$ is nonsingular and $p_{k}=\delta_{k} p_{k}^{P C G}$ for $\delta_{k}=\delta_{k}\left(\gamma_{k}\right)$.

Conversely, for any scalar $\delta_{k}$, except $\delta_{k}=0, \delta_{k}=\delta_{k}(1)$ and $\delta_{k}=1$, assume that $p_{k}=\delta_{k} p_{k}^{P C G}$ and assume that $B_{k}-B_{k-1}$ is symmetric and of rank one. Then, $B_{k}$ is a nonsingular matrix given by (26) for $\gamma_{k}=\gamma_{k}\left(\delta_{k}\right)$.

If, in addition, $B_{k-1}=B_{k-1}^{T} \succ 0$, then $B_{k}$ defined by (26) satisfies $B_{k} \succ 0$ if and only if $\gamma_{k}>1$ or $\hat{\gamma}_{k}<\gamma_{k}<0$, or equivalently if and only if $\gamma_{k}=\gamma_{k}\left(\delta_{k}\right)$ for $0<\delta_{k}<\delta_{k}(1)$ or $\delta_{k}>1$.

Finally, if $B_{i} p_{i}=-g_{i}, i=0, \ldots, k$, with $B_{0}=M$ and if, for $i=1, \ldots, k, B_{i-1}$ is updated to $B_{i}$ according to (26) for $\gamma_{i}$ such that $\gamma_{i} \neq 0, \gamma_{i} \neq \hat{\gamma}_{i}$ and $\gamma_{i} \neq 1$, then

$$
B_{k} p_{i}=\frac{\gamma_{i+1} \theta_{i}}{\gamma_{i+1}-1} H p_{i}, \quad i=0, \ldots, k-1
$$

\section{Conclusion}

In this paper we have derived necessary and sufficient conditions on the matrix $B_{k}$ in a QN-method such that $p_{k}$, obtained by solving $B_{k} p_{k}=-g_{k}$, satisfies $p_{k}=\delta_{k} p_{k}^{P C G}$ for some $\delta_{k} \neq 0$, where $p_{k}^{P C G}$ is the search direction of the preconditioned method of conjugate gradients. These conditions are stated in Proposition 4. The results have been derived for the case of CG and then extended to PCG for a symmetric positive definite preconditioning matrix $M$. 
Further, we have characterized the symmetric rank-one update matrices for QN that give parallel search directions to those of PCG. In Proposition 6, we show that the rank-one matrix must be a linear combination of $g_{k}$ and $g_{k-1}$, and also that almost any linear combination will do. In addition, we characterize the family of symmetric rank-one updates that preserve symmetry and positive definiteness of $B_{k-1}$.

Our focus is on the mathematical properties of PCG and QN in exact arithmetic. We want to stress that considering the numerical properties in finite precision is of utmost importance, but such an analysis is beyond the scope of this paper. See, e.g., [9] for an illustration of a case where PCG and QN generate identical iterates in exact arithmetic but the difference between numerically computed iterates for the two methods is large.

The results of the paper are meant to be useful as such, for understanding the behavior of exact linesearch quasi-Newton methods for minimizing a quadratic function. In addition, we hope that they can lead to further research on methods for unconstrained minimization. In particular, understanding the behavior of quasi-Newton methods on near-quadratic functions would be a subject of future research.

Acknowledgements We thank the editor and the anonymous referees for their constructive comments which significantly improved the presentation. Funding was provided by Vetenskapsrådet (Grant No. 6212014-4772).

Open Access This article is distributed under the terms of the Creative Commons Attribution 4.0 International License (http://creativecommons.org/licenses/by/4.0/), which permits unrestricted use, distribution, and reproduction in any medium, provided you give appropriate credit to the original author(s) and the source, provide a link to the Creative Commons license, and indicate if changes were made.

\section{References}

1. Davidon, W.C.: Variable metric method for minimization. SIAM J. Optim. 1(1), 1-17 (1991)

2. Demmel, J.W.: Applied Numerical Linear Algebra. Society for Industrial and Applied Mathematics (SIAM), Philadelphia (1997)

3. Fletcher, R.: Practical Methods of Optimization, 2nd edn. A Wiley-Interscience Publication. Wiley, Chichester (1987)

4. Fletcher, R., Powell, M.J.D.: A rapidly convergent descent method for minimization. Comput. J. 6, 163-168 (1963/1964)

5. Fletcher, R., Reeves, C.M.: Function minimization by conjugate gradients. Comput. J. 7, 149-154 (1964)

6. Forsgren, A., Odland, T.: On the connection between the conjugate gradient method and quasi-Newton methods on quadratic problems. Comput. Optim. Appl. 60(2), 377-392 (2015)

7. Gill, P.E., Murray, W., Wright, M.H.: Practical Optimization. Academic Press Inc. [Harcourt Brace Jovanovich Publishers], London (1981)

8. Golub, G.H., Van Loan, C.F.: Matrix Computations. Johns Hopkins Studies in the Mathematical Sciences, 3rd edn., pp. xxx+698. Johns Hopkins University Press, Baltimore, MD (1996)

9. Hager, W.W., Zhang, H.: The limited memory conjugate gradient method. SIAM J. Optim. 23(4), 2150-2168 (2013)

10. Hestenes, M.R., Stiefel, E.: Methods of conjugate gradients for solving linear systems. J. Res. Nat. Bur. Stand. 49(409-436), 1952 (1953)

11. Huang, H.Y.: Unified approach to quadratically convergent algorithms for function minimization. J. Optim. Theory Appl. 5, 405-423 (1970)

12. Kolda, T.G., O'Leary, D.P., Nazareth, L.: BFGS with update skipping and varying memory. SIAM J. Optim. 8(4), 1060-1083 (1998). electronic

13. Luenberger, D.G.: Linear and Nonlinear Programming, 2nd edn. Addison-Wesley Pub Co, Boston (1984) 
14. Nazareth, L.: A relationship between the BFGS and conjugate gradient algorithms and its implications for new algorithms. SIAM J. Numer. Anal. 16(5), 794-800 (1979)

15. Saad, Y.: Iterative Methods for Sparse Linear Systems, 2nd edn. Society for Industrial and Applied Mathematics, Philadelphia (2003)

16. Shewchuk, J.R.: An introduction to the conjugate gradient method without the agonizing pain. Technical report, Carnegie-Mellon University, Pittsburgh, PA, USA (1994) 